\title{
Caracterización de un sistema de adquisición de datos para un banco de prueba de Motor Diésel Monocilíndrico
}

\section{Characterization of a DAQ's system for a Single-cylinder Diesel Engine Test Bench}

DOI: http://dx.doi.org/10.17981/ingecuc.15.1.2019.14

Artículo de Investigación Científica. Fecha de Recepción: 07/10/2018. Fecha de Aceptación: 15/04/2019.

\author{
Jorge Duarte-Forero \\ Grupo KAI, Universidad del Atlántico, Barranquilla, (Colombia) \\ jorgeduarte@mail.uniatlantico.edu.co
}

Kevin Berrio-Orozco

Universidad del Atlántico, Barranquilla, (Colombia)

kmberrio@mail.uniatlantico.edu.co

\section{Ariel Guzmán-Fruto}

Universidad del Atlántico, Barranquilla, (Colombia) aguzmanf@mail.uniatlantico.edu.co

Para citar este artículo:

Jorge Duarte-Forero; Kevin Berrio-Orozco; Ariel Guzmán-Fruto "Caracterización de un Sistema de adquisición de datos para un Banco de Prueba de Motor Diésel Monocilíndrico”, INGE CUC, vol. 15, no. 1, pp. 155-167, 2019. DOI: http://doi.org/10.17981/ingecuc.15.1.2019.14

\section{Resumen}

Introducción- Dado el potencial investigativo que presentan actualmente los motores de combustión interna, una caracterización completa de sus condiciones de operación requiere tener en cuenta la variedad de los sensores utilizados para medir las magnitudes físicas, los múltiples dispositivos electrónicos usados para el acondicionamiento de señales y los estándares que denominan la sintaxis y sincronización de la comunicación. Por esta razón, se hace necesario el desarrollo de software como elemento de enlace del sistema de instrumentación, para proporcionar la visualización en tiempo real y almacenamiento de la información.

Objetivo- En este estudio se busca implementar una metodología para la selección y ajuste del sistema de adquisición de datos para un banco de prueba de motores Diesel, al igual que un software desarrollado para la visualización de las variables medidas por los instrumentos.

Metodología-Se realizó la selección de los instrumentos de medida empleados en el banco de pruebas, al igual que el diseño de los sistemas de acondicionamiento requeridos para cada instrumento, seguido de una puesta a punto y calibración. Paralelo a lo anterior, se elaboró un software interactivo en una plataforma de adquisición de datos para la visualización de los resultados obtenidos.

Resultados- Los resultados indican que la curva de calibración desarrollada produce un ajuste considerable al comportamiento de todos los datos, y mediante análisis de residuos se verifico que el modelo lineal determinado era el adecuado para describir el comportamiento de los sensores empleados.

Conclusiones- Se evidenció que 2 de los 7 sensores tenían valores de incertidumbre menores a la unidad de medida, lo que garantiza desviaciones pequeñas o prácticamente despreciables respecto al valor nominal. De los 7 tipos de instrumentos de medida implementados, el sensor correspondiente al régimen de giro presentó el valor más alto de incertidumbre; no obstante, este valor se ajusta a los errores permitidos en las mediciones de RPM que oscila un $5 \%$ su valor nominal.

Palabras Clave- Banco de Prueba, Caracterización, DAQ, Motor de Combustión Interna, Procesamiento de señales.

\begin{abstract}
Introduction- Given the research potential currently presented by internal combustion engines, a complete characterization of their operating conditions requires taking into account the variety of sensors used to measure their physical magnitudes, the multiple electronic devices used for the conditioning of signals and the standards that define the communication syntax and synchronization. For this reason, it is necessary to develop software as a link between the instrumentation system and the user, to provide real-time visualization and storage of information.
\end{abstract}

Objective- This study seeks to implement a methodology for the selection and adjustment of the data acquisition system for a Diesel engine test bench, as well as software developed to visualize the variables measured by the instruments.

Methodology- The selection of the instruments used in the test bench was carried out, as well as the design of the signal treatment devices for each instrument, followed by set-up and calibration. Parallel to the above, interactive software was developed in a DAQ's for the visualization of the results obtained with the acquisition system.

Results- The results indicate that the developed calibration curve produces a considerable fit of the experimental data, and using residue analysis it was verified that the linear model developed was adequate to describe the behavior of the sensors.

Conclusions- It was evidenced that 2 of the 7 of the sensors had uncertainty values lower than the measurement unit, which guarantees small or practically negligible deviations from the nominal value. Of the 7 types of instruments implemented, the sensor corresponding to the rotation speed presented the highest value of uncertainty; however, this value is adjusted to the errors allowed in the RPM measurements, which oscillates $5 \%$ of their nominal value.

Keywords- Test Bench, Characterization, DAQ, Internal Combustion Engine, Signal Processing. 


\section{INTRODUCCIÓN}

La gran evolución de los Motores de Combustión Interna (MCI) se atribuye a los diferentes estudios enfocados en el funcionamiento de estos. Diferentes trabajos, basados en la formulación de modelos termodinámicos para predecir la temperatura en el punto de ignición, la manipulación de la relación aire/combustible, ángulo del cigüeñal y presión de admisión, han permitido establecer estrategias de control para mejorar la operación en MCI [1], [2]. De igual manera, el uso de sistemas de Recirculación de Gases de Escape (EGR), la implementación de combustible gaseoso y el desarrollo de sistemas de postratamiento [3]-[6], han sido enfocados en disminuir la formación de contaminantes durante el proceso de combustión, aportando mejoras significativas al funcionamiento de estas máquinas.

Todos estos estudios descritos anteriormente, presentan una relación estrecha, dado que fueron desarrollados y validados en bancos de pruebas de motores, los cuales cuentan con robustos sistemas de instrumentación y adquisición de datos capaces de captar, almacenar y analizar las diferentes variables físicas medidas [7]. Asad et al. [8], destaca que, para la creación de un banco de pruebas para motores de combustión interna instrumentado adecuadamente, se debe medir: la presión interna del cilindro, temperaturas y presiones medias en los sistemas del motor, el flujo de combustible, el flujo de aire de admisión y la emisión de los gases de escape.

Teniendo en cuenta la variedad de los sensores utilizados para medir las magnitudes físicas, los múltiples dispositivos electrónicos usados para el acondicionamiento de señales y los estándares que denominan la sintaxis y sincronización de la comunicación, se hace necesario el desarrollo de software como elemento de enlace del sistema de instrumentación, para proporcionar la visualización en tiempo real y almacenamiento de la información.

A lo largo de presente manuscrito se detalla la cadena de medida implementada y la metodología aplicada para la consecución y validación de los resultados obtenidos.

\section{REVISIÓN DE LITERATURA}

La medición de temperatura juega un papel determinante para caracterizar el comportamiento en los MCI. Generalmente para captar esta variable es común el uso de termopares de baja capacitancia, a causa de su bajo costo y respuesta rápida [9]. [10]. De igual manera, la medición del flujo de aire de admisión es de suma importancia, dado que permite estimar parámetros como la relación aire/combustible y la recirculación de los gases de escape [8].

La medición de presión en subsistemas del motor, tal como sucede en la admisión de aire o gases de escape, es un procedimiento necesario para el diagnóstico de combustión. En efecto, Yao et al. [11], describe que la medida de presión en el colector de admisión permite controlar las condiciones termodinámicas del aire ingresado al motor. Por su parte Reyes et al. [12] resalta que la presión en la admisión debe ser medida con sensores piezoresistivos, que a diferencia de los piezoeléctricos usados para medir la presión en cámara, proporcionan el valor absoluto de la presión.

Por otra parte, el uso de una estructura de cadena de medida para el acondicionamiento de señal está definido por la necesidad especifica de cada sistema [13]. Existen casos en donde los parámetros deben ser medidos y registrados con altas frecuencias de muestreos; mientas que, en otros, los parámetros pueden ser medidos con frecuencias más bajas largas o incluso valores promedios [7]. Liu et al. [14] propone una metodología para el manejo de los datos, según la variable medida y la magnitud de la señal de salida de los sensores. El sistema de adquisición de datos desarrollado permite adquirir datos de alta frecuencia de muestreo como la señal de presión en cámara y bajas como temperaturas en la admisión configurando el equipo, bajo la premisa que se tenga disponible un numero suficientemente de canales para la adquisición de datos.

\section{III.Metodología de DESARRollo DE LA CADENA DE MEDIDA}

El sistema de instrumentación implementado en el banco de prueba de Motor Diésel, se desarrolló siguiendo la metodología mostrada en la Fig. 1. En su elaboración, se presentaron una serie de pasos intermedios, los cuales se describen a continuación.

\section{A. Selección de Instrumentos de medida}

Fundamentados en antecedentes relacionados a temáticas de MCI y aplicaciones de instrumentos de medición, se definieron las magnitudes físicas de temperatura, presión, flujo másico, régimen de giro y potencia eléctrica, como las principales variables a medir en el banco de prueba. Los instrumentos de medida fueron seleccionados teniendo en cuenta principios de funcionamiento y condiciones de operación a los que estarían expuestos, según la ubicación en los diferentes subsistemas del motor, como se observa en la tabla 1. 


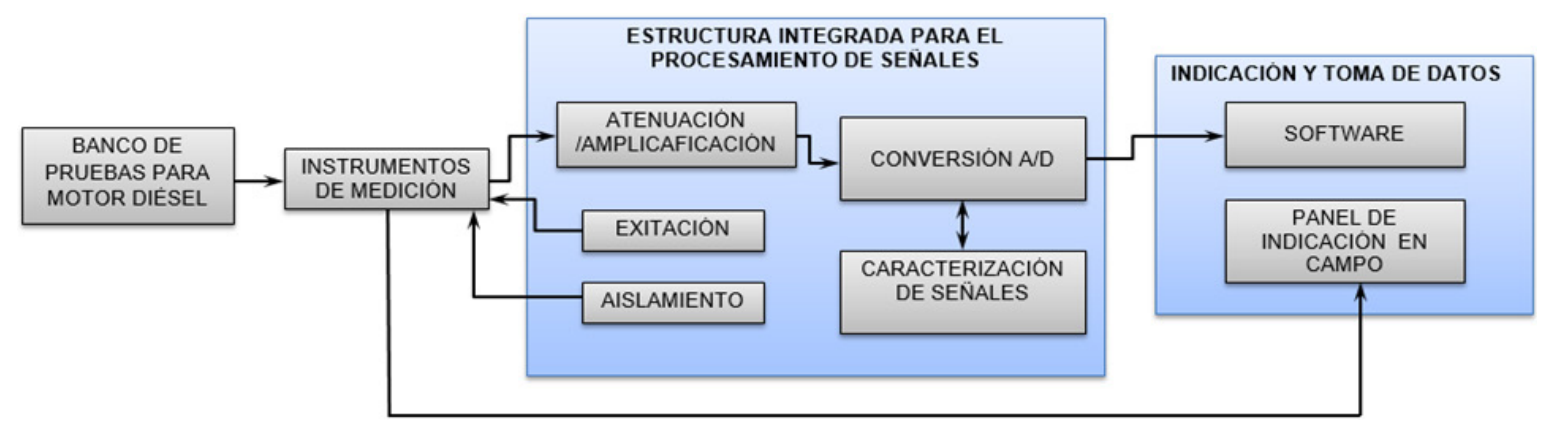

Fig. 1. Esquema de la cadena de medida implementada en banco de prueba de motor Diésel. Fuente: Autores.

Tabla 1. Instrumentos de Medida Implementados en Banco de Prueba de Motor Diesel.

\begin{tabular}{|c|c|c|}
\hline Variable & Sensor & Ubicación \\
\hline \multirow{2}{*}{ Temperatura } & & Colector de Aire de Admisión \\
\cline { 2 - 3 } & \multirow{2}{*}{ Termopar Tipo K } & Colector de Gases de Escape \\
\cline { 2 - 3 } & & Superficie de Culata del Motor \\
\cline { 2 - 3 } & & Reservorio de Combustible \\
\cline { 2 - 3 } & Termostato DHT22 & Ambiente \\
\hline \multirow{2}{*}{ Presión } & Sensor PSACO1 Piezo-resistivo & Colector de Aire de Admisión \\
\hline Flujo Másico & Sensor BOSCH Hilo Caliente & Colector de Aire de Admisión \\
\hline Régimen de Giro & Sensor de Proximidad Efecto Hall & Volante del Motor \\
\hline Humedad Relativa & Sensor Capacitivo DHT22 & Ambiente \\
\hline Presión Atmosférica & Barómetro Digital Bosch BPM180 & Ambiente \\
\hline Intensidad (Corriente) & Sensor de Efecto Hall SCT-013000 & Salida del Generador \\
\hline
\end{tabular}

Fuente: Autores

Tabla 2. Requerimientos de Acondicionamiento de Señal de Sensores Implementados. AP (APLiCA), NA (No ApliCA).

\begin{tabular}{|c|c|c|c|c|c|c|c|}
\cline { 2 - 9 } \multicolumn{1}{c|}{} & \multicolumn{9}{c|}{ Acondicionamiento de Señal } \\
\cline { 2 - 9 } & Amplificación & Atenuación & Aislamiento & Filtrado & Excitación & Linealización & Conversión \\
\hline Temperatura & AP & NA & AP & NA & NA & AP & AP \\
\hline Flujo Másico & NA & NA & AP & NA & AP & AP & AP \\
\hline Presión & NA & NA & AP & NA & AP & AP & AP \\
\hline Régimen de Giro & NA & AP & AP & NA & AP & AP & AP \\
\hline $\begin{array}{c}\text { Intensidad de } \\
\text { Corriente }\end{array}$ & AP & NA & AP & NA & AP & AP & AP \\
\hline $\begin{array}{c}\text { Condiciones } \\
\text { Ambientales }\end{array}$ & NA & NA & AP & NA & AP & AP & AP \\
\hline
\end{tabular}

Fuente: Autores.

\section{B. Estructura integrada para el procesamiento de señales}

Acondicionar una señal conduce a ejecutar etapas de modificación de los niveles de la señal, linealización, filtrado de la señal y conversión de la señal [15]. Cada una de estas etapas, son llevadas a cabo por uno o varios dispositivos electrónicos, ya sea individual o conjuntamente. La tabla 2 , presenta un resumen de los tipos de acondicionamiento de señales aplicados a cada uno de los instrumentos de medida seleccionados.

Teniendo presente los requerimientos de los instrumentos seleccionados y la necesidad de adquirir la información de una manera confiable, se implementaron Arduinos Mega 2560 como tarjeta de adquisición datos. Estos actúan como interfaz entre las señales enviadas por los sensores y recibidas por el ordenador. 


\section{1) Acondicionamiento de señal de Termopares}

Para obtener la señal de temperatura medida en los diferentes puntos del motor, se utilizó el MAX6675, que consiste en un circuito integrado para amplificar, compensar y digitalizar la señal generada por la termocupla. Este dispositivo proporciona una señal acondicionada a la Tarjeta Arduino a través de una interfaz SPI (Serial Peripheral Interface) de solo lectura.

\section{2) Acondicionamiento de señal de medidor de flujo másico}

El caudalímetro de hilo caliente Bosch 22680-7J600, es un transmisor análogo, compuesto de un dispositivo electrónico de alta precisión que procesa y convierte la señal generada a un formato estándar de tensión. Para esto, el sensor requiere de una etapa de excitación generada por una fuente de alimentación de 12VDC y una fuente de alimentación de 5 VDC para la resistencia de referencia. Adicional a esto, se requiere un proceso de aislamiento, con el fin de proteger la señal emitida de perturbaciones externas; y un proceso de digitalización de la señal, llevada a cabo por la Tarjeta Arduino.

\section{3) Acondicionamiento de señal del sensor de presión}

El sensor-indicador de presión PSA-CO1 es un transductor piezo-resistivo que proporciona una señal análoga de niveles acordes a la Tarjeta Arduino. Al sensor solo le fueron aplicadas etapas de excitación generada por una fuente de $12 \mathrm{VDC}$, un proceso de aislamiento para salvaguardar la señal transmitida y una etapa de conversión realizado por la tarjeta Arduino, lo cual se muestra en la Fig. 2.

\section{4) Acondicionamiento de señal del sensor de régimen de giro}

Para captar la magnitud de velocidad de giro, se dispuso de un transductor de proximidad magnético NJK-5002C que ofrece una señal de salida analógica proporcional al campo magnético generado al momento de captar el objeto de detección. Esta tensión de salida requirió un acondicionamiento compuesto por una etapa de excitación del sensor, el cual fue generada por una fuente de alimentación, una etapa de atenuación llevada a cabo por un circuito divisor de voltaje, un proceso de aislamiento, y una etapa de digitalización realizado por la Tarjeta Arduino.

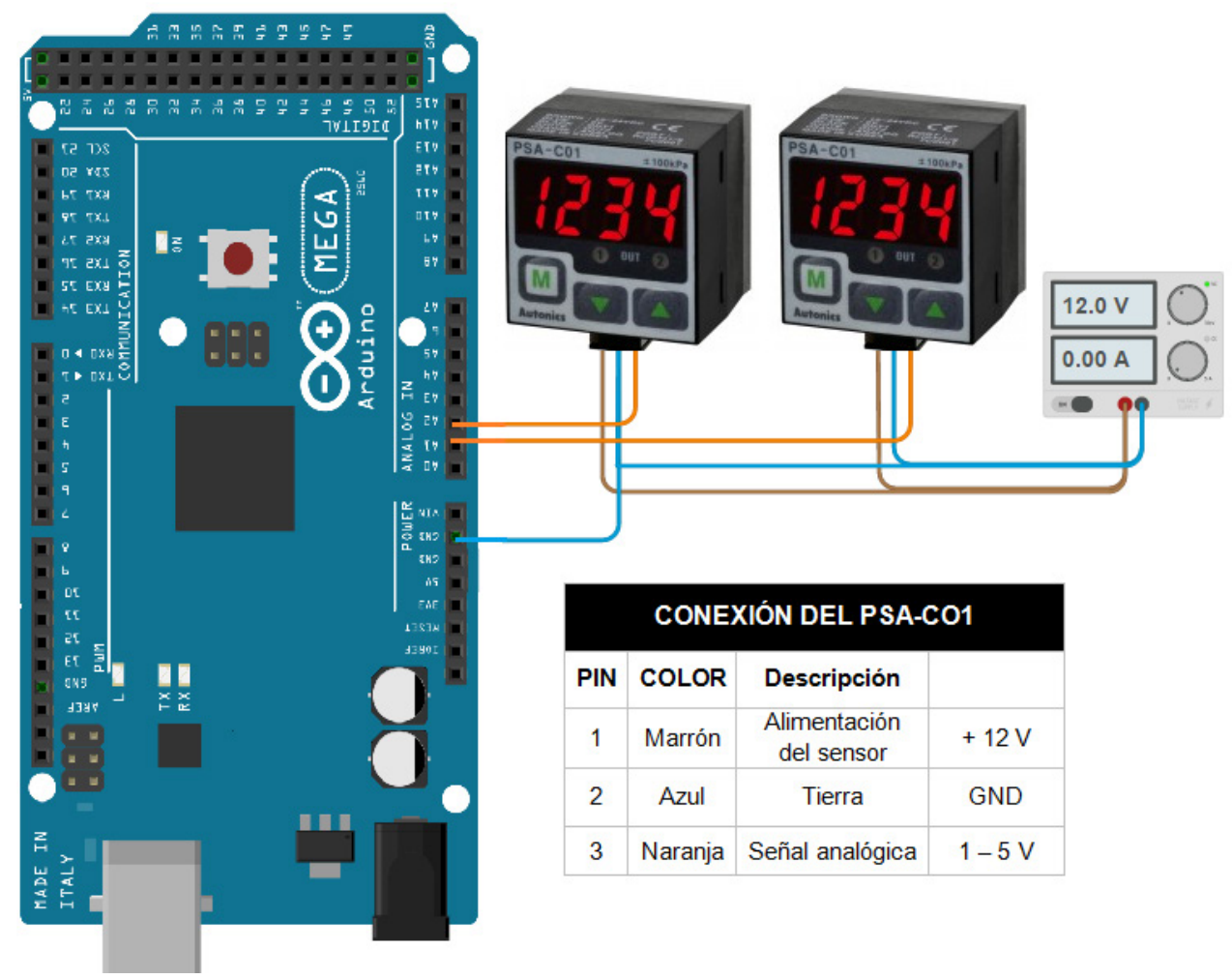

Fig. 2. Esquema de conexión del sensor PSA-CO1 a la tarjeta Arduino. Fuente: Autores. 


\section{5) Acondicionamiento de señal de los sensores de condiciones ambientales}

Para medir la magnitud de humedad relativa y temperatura ambiente se dispuso el sensor DHT22, compuesto de una hilera de empaquetado con cuatro pines distribuidos para su alimentación y emisión de señal de salida de tipo PWM (Pulse Width Modulation). A este sensor le fueron aplicados etapas de excitación, aislamiento en las conexiones y conversión para el proceso de acondicionamiento de señal.

La magnitud de presión atmosférica fue determinada mediante el sensor Bosch BPM180, quien cuenta con un circuito integrado $\mathrm{I}^{2} \mathrm{C}$ (Inter Integrated Circuit), compatible con la Tarjeta Arduino. Este sensor se compone de cuatro puertos distribuidos para su alimentación y salida de señal, los cuales fueron conectados a la Tarjeta Arduino en donde se realizó el proceso de conversión y linealización.

\section{6) Acondicionamiento de señal del sensor de corriente}

La salida del sensor de corriente SCT-013-000 es una señal eléctrica de tipo alterna, cuyos valores no están dentro del rango de entradas analógicas de la tarjeta Arduino. Para obtener una salida acorde con los niveles de esta, fue necesario rectificar y amplificar la señal proveniente del sensor. Para esto, se utilizó un amplificador operacional LM358, encargado de realizar el acondicionamiento sin pérdidas de tensión en la señal emitida.

En este orden de ideas, al haber definido e implementado los diferentes dispositivos de acondicionamiento de señal, se diseñó una tarjeta electrónica (tal como se ilustra en la Fig. 3) para agrupar dichos requerimientos y en conjunto con las tarjetas Arduino Mega, se logró proporcionar una estructura integrada para el procesamiento de señales del banco de prueba.

\section{Proceso de caracterización de señales}

Parte del proceso de acondicionamiento se ve ligado a la aplicación de alguna metodología para interpretar la señal procedente del sensor como una medición física. Esto puede ser llevado a cabo ya sea a través de dispositivos electrónicos, métodos numéricos en el sistema de adquisición o mediante un proceso de caracterización. Esta última consiste en la búsqueda de la ecuación característica que determina la razón de cambio de la variable física medida, respecto a una señal de salida generada por el sensor.

El proceso de caracterización ofrece un factor determinante para la interpolación de valores correspondientes a la magnitud física. Esta metodología, fue aplicada a los sensores de presión y flujo másico implementados en el banco, como una de las etapas de acondicionamiento requeridas por estos instrumentos.

\section{1) Caracterización del sensor de presión}

Para caracterizar la señal del sensor de presión utilizado para medir la presión promedio en la admisión y en escape, se realizó el montaje del sistema de carga del sensor (Fig. 4), comprendido por un mecanismo embolo-cilindro y una manguera neumática conectada al sensor y sellada de tal manera que no presente fugas en el sistema.

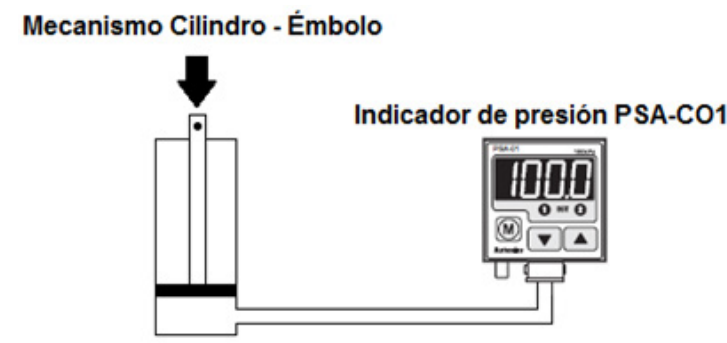

Fig. 4. Sistema de generación de carga del sensor PSA-CO1. Fuente: Autores.

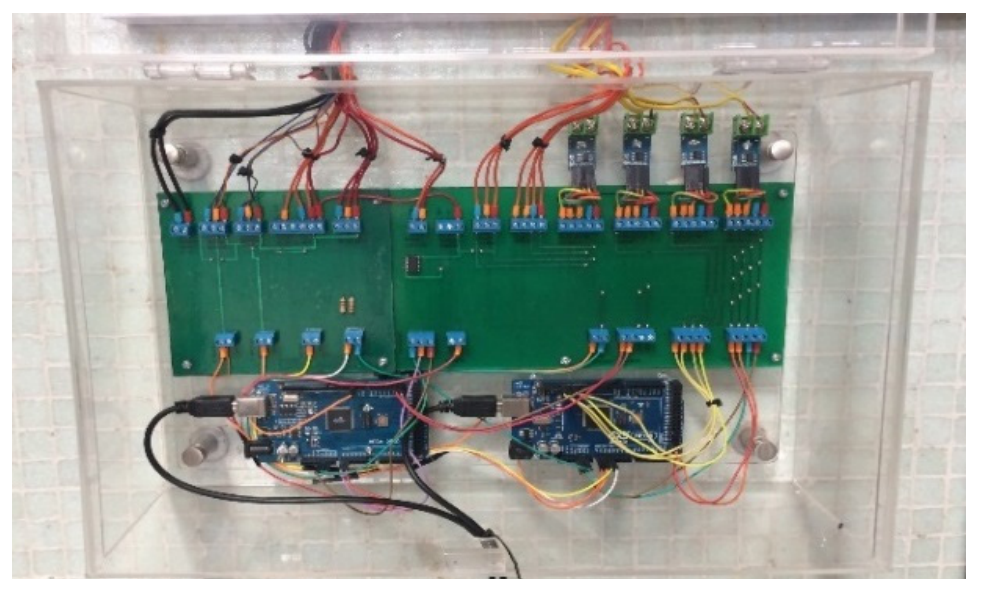

Fig. 3. Estructura integrada para el procesamiento de señales. Fuente: Autores. 
Al ejercer una carga sobre el mecanismo, se generó presión, siendo medida e indicada por el sensor PSA-CO1. Manteniendo la carga constante durante cierto intervalo de tiempo, la señal de tensión generada fue adquirida con la ayuda de la estructura diseñada para el procesamiento de señales, logrando de esta forma determinar y almacenar la relación de la magnitud mostrada por el indicador del sensor y la señal de tensión emitida por el mismo. Los datos fueron adquiridos durante un lapso de tiempo de 25 segundos, con una frecuencia de adquisición de 1 segundo para las diferentes condiciones de cargas establecidas.

Con la información obtenida, se realizó un análisis de datos para encontrar el comportamiento del sensor PSA-CO1. Se determinaron las medidas de tendencia central, escogiendo la moda como dato representativo dentro del intervalo de valores adquiridos para cada medida de presión, permitiendo de igual manera descartar los datos erróneos ocasionados por ruidos de la señal durante la etapa de medición. Tomando en cuenta las especificaciones del fabricante, se determinó que la señal generada por el transductor de presión es de carácter lineal en relación a la magnitud de presión, por lo que su ecuación característica se puede expresar como:

$$
y=a+b \cdot x
$$

Donde y es la variable dependiente y representa el valor de la magnitud de presión; $x$ es la variable independiente y representa el valor de la señal tensión generada por el sensor; $a$ es la pendiente de la recta y $b$ la ordenada al origen. Mediante un análisis por regresión lineal, se determinó el valor de los coeficientes $a$ y $b$ de tal forma que se ajusten al comportamiento de toda la muestra.

\section{2) Caracterización del sensor del flujo másico}

Para caracterizar la señal del sensor de flujo másico Bosch 22680-7J600, se tomó como instrumento de referencia al caudalímetro FLUKE 922 (cuyo esquema básico se ilustra en la Fig. 5), quien proporciona la velocidad del flujo de aire, bajo el principio de presión diferencial obtenida de la medición de tubo de Pitot que lo conforma.

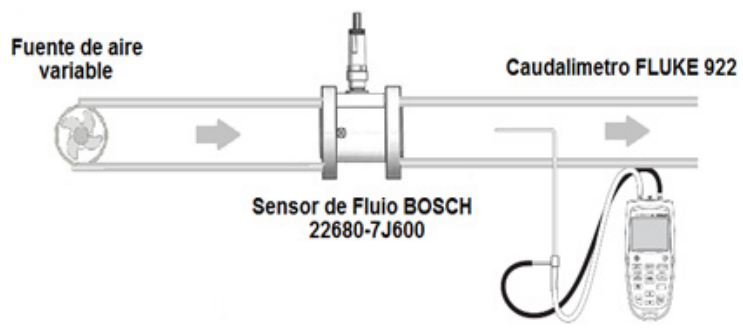

Fig. 5. Montaje de sensor BOSCH 22680-7J600 y caudalímetro FLUKE 922. Fuente: Autores.
Para la prueba de caracterización se realizó la instalación de ambos sensores de flujo a un ducto de $6 \mathrm{~cm}$ de diámetro y $3 \mathrm{~m}$ de longitud, garantizando que el flujo se desarrolle. El sensor BOSCH 22680-7J600 fue ubicado a una distancia de $1,5 \mathrm{~m}$ de la parte inicial del ducto, garantizando el desarrollo del flujo emitido por la fuente de aire y el caudalímetro FLUKE 922 a $1 \mathrm{~m}$ de distancia del sensor BOSCH 22680-7J600, asegurando de igual manera un perfil de velocidad del flujo constante.

Durante el proceso se establecieron velocidades de flujo constante en base a la medida arrojada por el caudalímetro FLUKE. Manteniendo la condición de flujo durante un lapso de tiempo de $1 \mathrm{~min}$, fueron almacenadas con una frecuencia de adquisición de 1 $\mathrm{s}$, las señales de tensión generadas por el sensor de hilo caliente BOSCH.

Posterior a esto se realizó un análisis de datos para encontrar el comportamiento particular del sensor $\mathrm{BOSCH}$, frente a los valores de velocidad indicados por el caudalímetro FLUKE. Con (2) se determinó el flujo volumétrico (Q) equivalente a la velocidad indicada por el caudalímetro FLUKE:

$$
Q=A \cdot v
$$

Siendo $A$ el área del ducto de circulación del fluido y $v$ la velocidad del fluido.

Igual que en el proceso de caracterización del sensor de presión PSA-CO1, fueron calculadas las medidas de tendencia central, seleccionando la moda como dato representativo del grupo de valores adquiridos para cada magnitud de velocidad, descartando los datos equívocos atribuidos a las perturbaciones durante el proceso de medición. A partir de información suministrada por el fabricante, el sensor de flujo BOSCH 22680-7J600 describe un comportamiento polinomial, por lo que se adoptó una función polinomial de orden cuatro para garantizar el ajuste de todos los datos.

$$
y=a x^{4}+b x^{3}+c x^{2}+d x+e
$$

Donde $y$ es la variable dependiente y representa el valor de la magnitud flujo volumétrico; $x$ es la variable independiente y representa el valor de la señal tensión generada por el sensor BOSCH; $a, b, c, d$ y $e$ los coeficientes de la ecuación determinados mediante un análisis de regresión lineal múltiple.

\section{Software Desarrollado}

Como elemento final de la cadena de medida se diseñó el software SI-MCI2017 (cuya estructura se muestra a continuación en la Fig. 6), bajo los entornos de programación LabVIEW ${ }^{\mathrm{TM}}$ y Arduino ${ }^{\mathbb{}}$, orientado a la aplicación de monitoreo de los instrumentos de medición [16]. Para el desarrollo del programa, se utilizó la herramienta de interfaz LINX MakerHub, quien permitió la conexión y adquisición con la plataforma embebida Arduino ${ }^{\circledR}$. Gracias a los 
firmwares que esta interface ofrece, se logró tener acceso a señales digitales, analógicas, PWM y SPI con la Tarjeta de Arduino ${ }^{\circledR}$.

\section{1) Programación para Visualización de variables}

Para visualizar la magnitud de los instrumentos de medida se desarrollaron diferentes códigos que contienen los firmwares y herramientas necesarias para la conexión, lectura y procesamiento de las señales.

\section{- Código de visualización Temperatura}

En este código se establecieron las herramientas necesarias para la comunicación SPI con el programa, llevando a cabo la transformación de la señal recibida a un valor de temperatura equivalente mediante un grupo de operaciones binarias. Además, se determinó una constante de compensación para cada uno de las magnitudes de temperatura visualizadas, garantizando una medida exacta. La Fig. 7 ilustra las características del código mencionado.

\section{- Código de visualización Presión}

Para obtener el valor de la magnitud de presión, se hizo uso de la ecuación determinada en el proceso de caracterización del sensor de presión PSA-CO1 en el código desarrollado, mediante una herramienta de $L a b V I E W^{T M}$ encargada de evaluar la señal adquirida y mostrar un valor equivalente de presión.

\section{- Código de flujo másico}

En el desarrollo del código de visualización de flujo másico, se introdujo inicialmente la ecuación característica del sensor Bosch para determinar el flujo volumétrico de aire equivalente a la señal emitida por el sensor. Luego, mediante una interpolación realizada por herramientas de $L a b V I E W^{T M}$, se determinó la densidad del aire equivalente a la temperatura medida por el sensor DHT22. A partir del flujo volumétrico y la densidad equivalente se obtuvo el valor correspondiente al flujo másico de aire.

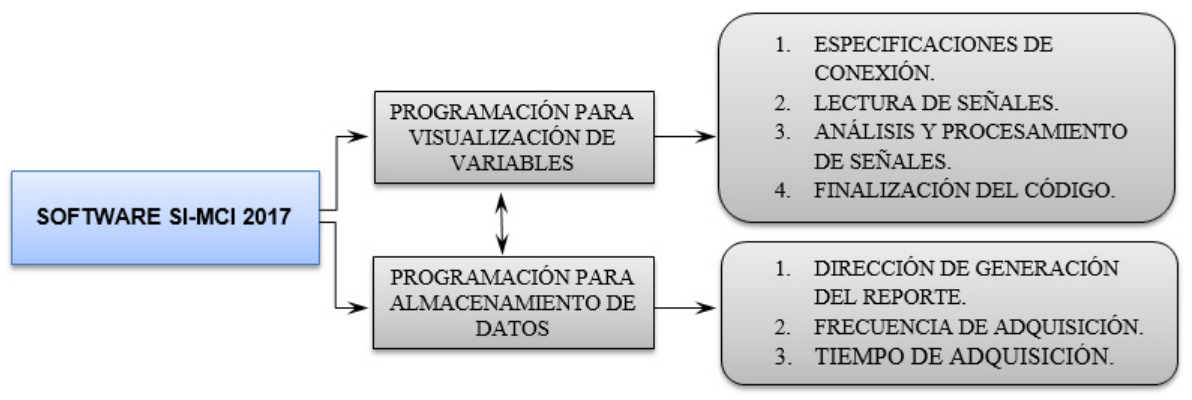

Fig. 6. Estructura del Software desarrollado. Fuente: Autores.

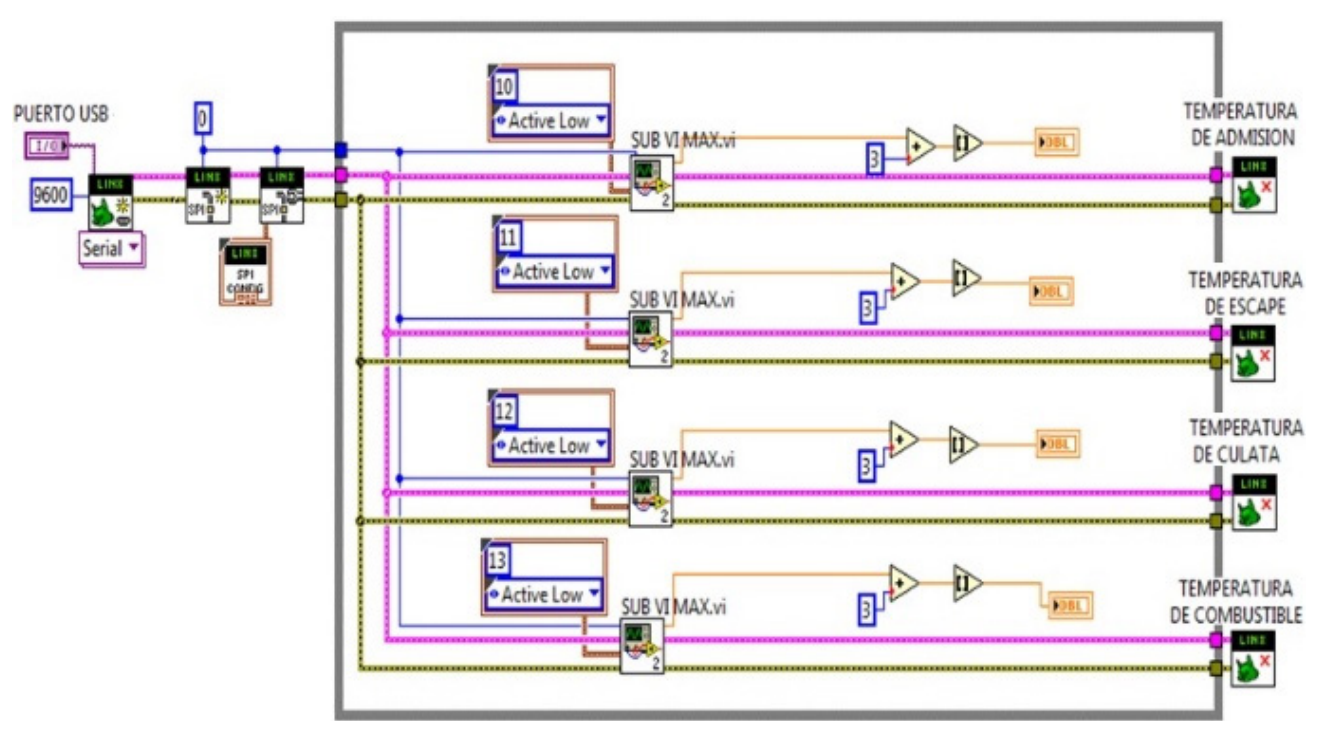

Fig. 7. Código para la visualización de temperatura. Fuente: Autores. 


\section{- Código de régimen de Giro}

Para determinar el Régimen de Giro del Motor (RPM) fue necesario ejecutar varios pasos secuenciales en el código, con el objetivo de calcular la magnitud de velocidad durante un intervalo de tiempo. En el desarrollo se implementó una metodología de monitoreo de frecuencia de pulsos, en la que se realizó un conteo de las señales que representan la detección del objeto ferromagnético, durante un intervalo de tiempo establecido de $5 \mathrm{~s}$. Teniendo en cuanta que cada pulso emitido por el sensor NJK-5002C representa una revolución del eje del motor, se determina la magnitud medida a partir de (4).

$$
\text { Régimen de Giro }=\frac{\# \text { pulsos }}{5 s} \cdot \frac{60 s}{1 \min } \mathrm{RPM}
$$

\section{- Código de intensidad de corriente} $y$ condiciones ambientales

Para la visualización de las variables de condiciones ambientales y la medida de intensidad de corriente se hizo uso de la segunda Tarjeta Arduino
Mega, debido a que la etapa de procesamiento de estas señales fue ejecutada a través del entorno de programación Arduino ${ }^{\circledast}$. Para esto se desarrolló un código integrando la sintaxis necesaria para interpretar la señal emitida por los sensores. Sin embargo, para unificar la visualización de estas variables en el software desarrollado, se estableció una interfaz de comunicación con LabVIEW $W^{T M}$.

\section{2) Programación para almacenamiento de datos}

La programación diseñada para el almacenamiento de datos consistió en un código desarrollado para ser ejecutado cuando así lo determine el operador, el cual se muestra en la Fig. 8. El código comprende las funciones de control sobre el tiempo de adquisición, la frecuencia de adquisición de datos y la dirección del reporte donde se genera el almacenamiento de la información. Al ser activa la adquisición de datos, el programa toma los valores correspondientes de las magnitudes físicas visualizadas y genera un reporte de datos al finalizar el tiempo de adquisición establecido.

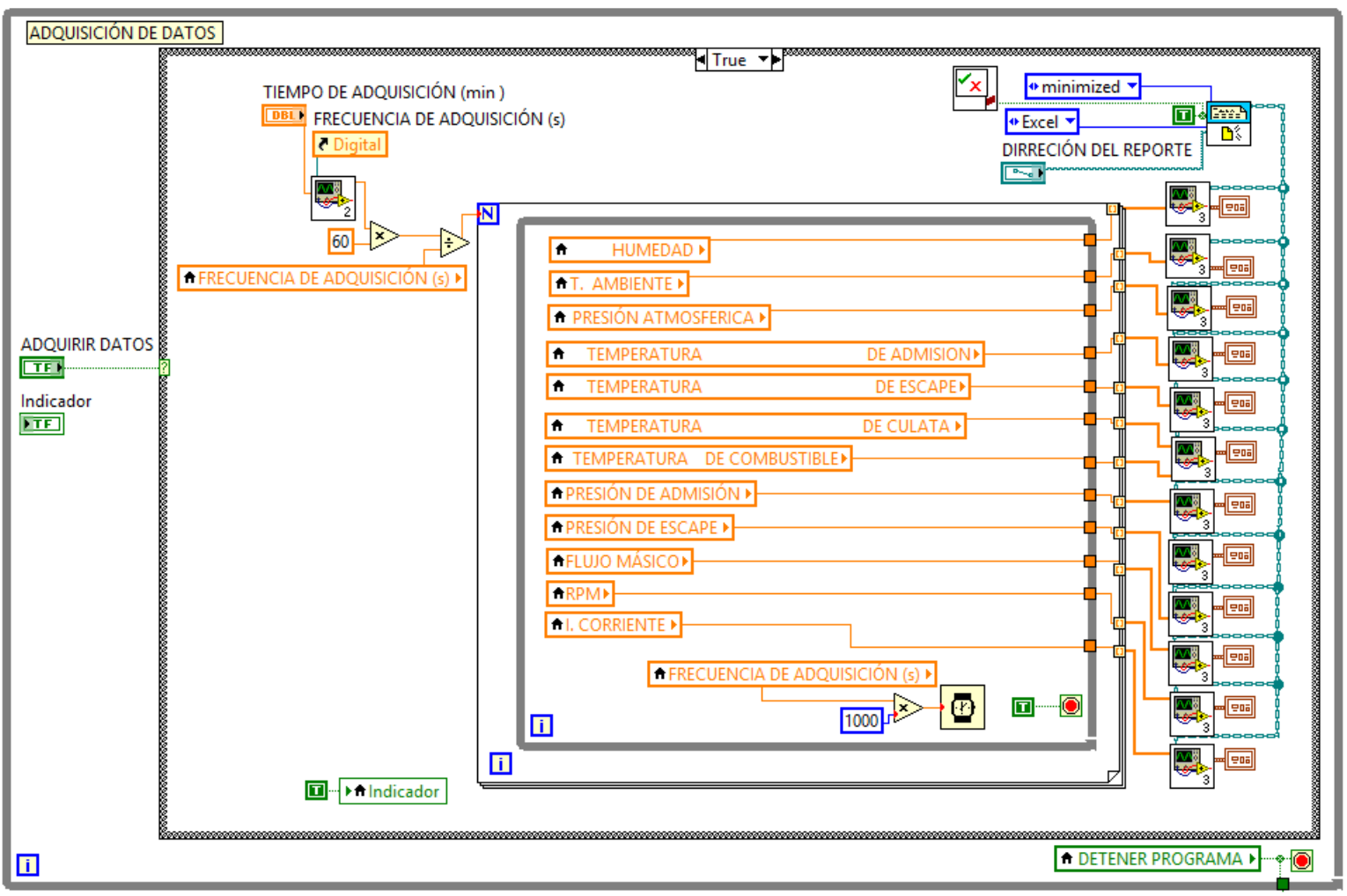

Fig. 8. Código para el almacenamiento de datos. Fuente: Autores. 


\section{B. Análisis de incertidumbre de los sensores implementados}

La operación del sistema de instrumentación puede estar ligada a errores en las medidas, los cuales son atribuidos a la variabilidad natural de la magnitud medida, características de los instrumentos de medición y condiciones en las que se está midiendo. Es por esto que, a los instrumentos de medida implementados en el banco, les fue determinado un valor de incertidumbre, encargado de caracterizar la dispersión de los valores que pueden ser atribuidos razonablemente a la magnitud medida.

Según la Guía para la Expresión de la Incertidumbre de Medida (GUM) se puede agrupar los componentes de incertidumbre es dos categorías de evaluación: Tipo A y Tipo B. La primera estima una distribución en base a mediciones repetidas obtenidas del mismo proceso de medición y la segunda, determina una distribución en base a experiencia o información externa. Sin embargo, esta categorización no implica que exista alguna diferencia de naturaleza de los componentes resultantes de cada uno estos tipos, sino en dos formas diferentes de evaluar los componentes de incertidumbre, basándose en distribuciones de probabilidad.

Por lo anterior, se adoptó una metodología de evaluación de incertidumbre tipo B, asociada a errores de resolución y precisión de los instrumentos [17]. En este tipo de evaluación, la incertidumbre de medición se sustenta en información externa u obtenida empíricamente, tales como: certificados de calibración, resultados de medidas anteriores, manuales de los instrumentos de medición, especificación del fabricante, valores de referencia de libros y resultados de investigación. De (5) y (6) se determinó la fuente de incertidumbre asociada a la exactitud y precisión de los instrumentos, siendo EMP el error máximo permisible.

$$
\begin{gathered}
u_{1 B}(p)=\frac{E M P}{\sqrt{3}}(5) \\
E M P=\frac{\text { Clase } \cdot \text { Intervalo de Medida }}{100}
\end{gathered}
$$

Por otra parte, una de las fuentes de incertidumbre de los instrumentos es la resolución de su dispositivo indicador. Tratándose de un instrumento digital con resolución $v_{d}$ el valor de señal de entrada que produce cierta indicación puede ubicarse con igual probabilidad en el intervalo medio de la resolución:

$$
u_{2 B}(p)=\frac{\frac{V d}{2}}{\sqrt{3}}(7)
$$

De esta manera, el valor de incertidumbre combinada atribuido a cierta medición $u_{c}(p)$, de los instrumentos de medida fue determinada a partir de la ecuación

$$
u_{c}(p)=\sqrt{\left(u_{1 B}\right)^{2}+\left(u_{2 B}\right)^{2}}
$$

Buscando aumentar la probabilidad hasta valores más confiables, se multiplica la incertidumbre combinada por una constante $K$, denomina factor de cobertura, determinado a partir de un nivel de confianza $p$ establecido:

$$
U_{p}=K \cdot u_{c}
$$

Siendo, $U_{p}$ el valor de incertidumbre expandida que define un intervalo de confianza en torno al resultado de una medición.

A partir de una distribución rectangular se determinó el factor de cobertura $K$ para un nivel de confianza establecido.

$$
\beta=\frac{\left|\mathrm{A}_{1}-\mathrm{A}_{2}\right|}{\mathrm{A}_{1}+\mathrm{A}_{2}}
$$

$$
\text { Donde } A_{1}=E M P \text { y } A_{2}=\frac{v d}{2}
$$

$k(p)=\frac{1}{\sqrt{\frac{1+\beta^{2}}{6}}} \cdot \frac{p(1+\beta)}{2} \leftrightarrow \frac{p}{2-p}<\beta$

$k(p)=\frac{1}{\sqrt{\frac{1+\beta^{2}}{6}}} \cdot\left(1-\sqrt{(1-p) \cdot\left(1-\beta^{2}\right)}\right) \leftrightarrow \beta \leq \frac{p}{2-p}(12)$

\section{III.Resultados y ANÁlisis}

En el proceso de caracterización del sensor de presión PSA-CO1, se graficaron los valores representativos de cada señal de tensión adquirida respecto a la magnitud de presión correspondiente, evidenciando un comportamiento lineal de la curva características del sensor PSA-CO1, que coincide con la información suministrada en el catálogo del fabricante. Aplicando un análisis por regresión lineal, se determina el valor de los coeficientes $a$ y $b$, lo que se muestra en la tabla 3 .

Tabla 3. Análisis de Regresión Lineal.

\begin{tabular}{|c|c|c|}
\hline \multicolumn{3}{|c|}{ Regresión Lineal } \\
\hline Coeficiente de Determinación & $\mathrm{R}^{2}$ & $99,973 \%$ \\
\hline \multirow{2}{*}{ Coeficientes } & $\mathrm{a}$ & $-145,3955$ \\
\cline { 2 - 3 } & $\mathrm{b}$ & 49,2205 \\
\hline
\end{tabular}

Fuente: Autores. 
Por el coeficiente de determinación $\left(R^{2}\right)$, se pudo inferir que la ecuación supuesta se ajustaba al comportamiento de la mayoría de los datos, concluyendo que al menos el 99,97\% de la variabilidad de los factores de corrección se explica a través de las variables de regresión. Sin embargo, realizando un análisis de residuos se verifico que el modelo lineal determinado era el adecuado para describir el comportamiento del sensor PSA-CO1 [18], lo cual coincide con el ajuste observado en la gráfica de los residuos de la Fig. 9.

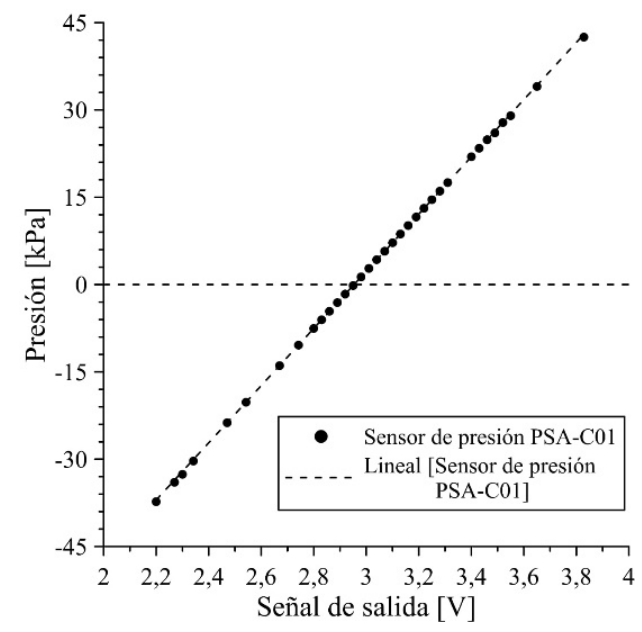

Fig. 9. Comportamiento de sensor de presión PSA-CO1. Fuente: Autores.

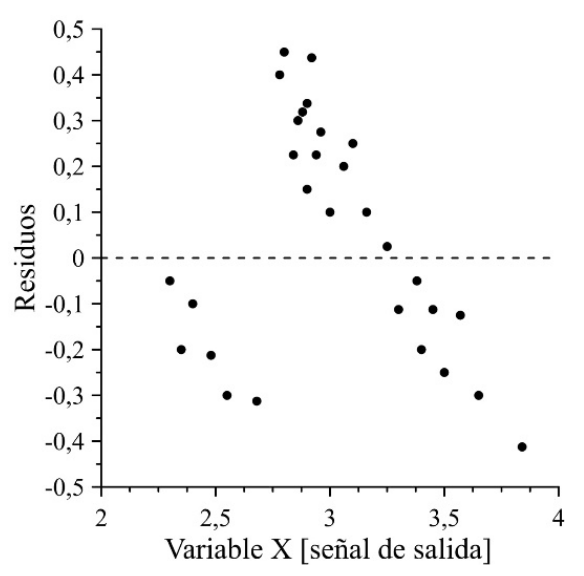

Fig. 10. Gráfico de residuales.

$$
\text { Fuente: Autores. }
$$

La Fig. 10 representa los residuos frente a los valores estimados de la variable independiente (señal de salida del sensor de presión PSA-CO1). En ella se evidencia que los residuos se dispersan de forma homogénea, no describen ninguna forma funcional y oscilan en una banda alrededor de cero. Por tal razón, (13) representa de manera apropiada la razón de cambio de la variable física medida respecto a una señal de salida generada por el sensor de presión PSA-CO1.

$$
\mathrm{Y}=-145,3955+49,2205 \cdot X
$$

En la Fig. 11 se presenta el valor representativo de la señal de tensión generada por el sensor BOSCH 22680-7J60 en función del flujo volumétrico.

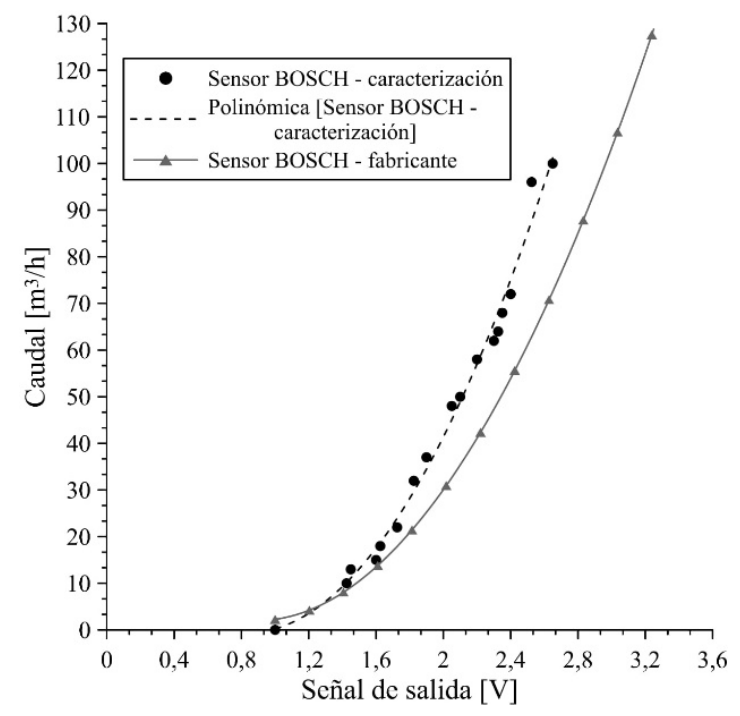

Fig. 11. Curva del fabricante y curva obtenida del proceso de caracterización del sensor de flujo de aire BOSCH 22680-7J600. Fuente: Autores.

A partir de la información gráfica, suministrada en el catálogo del fabricante, el sensor de flujo BOSCH 22680-7J600, describe un comportamiento polinomial. Aplicando un análisis por regresión lineal múltiple, se han determinado los coeficientes de la función que mejor se ajusta al comportamiento de las variables, tal como se presenta en la tabla 4 . En este caso, se indica que el 99,80\% de la variabilidad de los factores es explicada por la regresión.

Tabla 4. Análisis de Regresión Lineal Múltiple.

\begin{tabular}{|c|c|c|}
\hline \multicolumn{3}{|c|}{ Regresión Lineal Múltiple } \\
\hline Coeficiente de Determinación & $\mathrm{R}^{2}$ & $99,80 \%$ \\
\hline \multirow{2}{*}{ Coeficientes } & $\mathrm{e}$ & 189,48 \\
\cline { 2 - 3 } & $\mathrm{d}$ & $-463,52$ \\
\cline { 2 - 3 } & $\mathrm{c}$ & 388,29 \\
\cline { 2 - 3 } & $\mathrm{b}$ & $-130,85$ \\
\cline { 2 - 3 } & $\mathrm{a}$ & 17,15 \\
\hline
\end{tabular}

Fuente: Autores

A partir de los resultados, graficando los residuos frente a cada uno de los grados de la variable $X$, se han ilustrado los siguientes gráficos que se integran en la Fig. 12. 

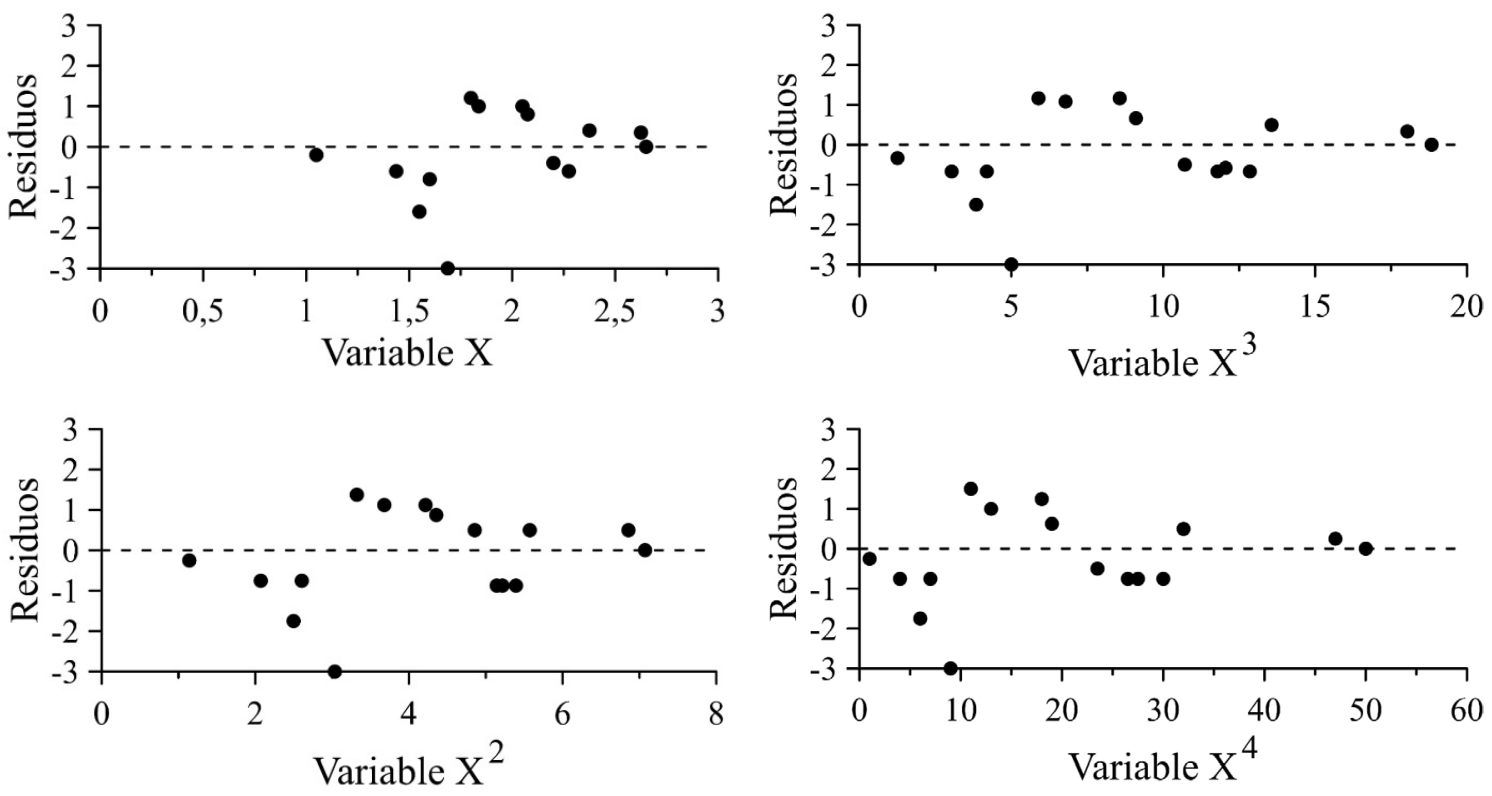

Fig. 12. Gráfico de residuales de la regresión lineal múltiple. Fuente: Autores.

Tabla 5. Parámetros de los Sensores e Indicadores Implementados en el Banco de Prueba del Motor Diésel.

\begin{tabular}{|c|c|c|c|c|c|}
\hline Variable & Tipo de Sensor & Clase & Rango & Span & Resolución \\
\hline Temperatura & Termopar Tipo K & $0,1 \%$ & {$[-200 \text { a } 1370]^{\circ} \mathrm{C}$} & $1570^{\circ} \mathrm{C}$ & $1^{\circ} \mathrm{C}$ \\
\hline Presión & Piezorresistivo & $0,3 \%$ & {$[-100$ a 100$] \mathrm{kPa}$} & $200 \mathrm{kPa}$ & $0,2 \mathrm{kPa}$ \\
\hline Flujo Másico & Hilo Caliente & $0,5 \%$ & {$[0$ a 450$] \mathrm{kg} / \mathrm{h}$} & $450 \mathrm{~kg} / \mathrm{h}$ & $0,1 \mathrm{~kg} / \mathrm{h}$ \\
\hline Régimen de Giro & Efecto Hall & $0,03 \%$ & [5 a 9999] RPM & 9994 RPM & $1 \mathrm{RPM}$ \\
\hline Humedad Relativa & Capacitivo & $2 \%$ & [0 a 100] \%HR & $100 \% \mathrm{HR}$ & $1 \% \mathrm{HR}$ \\
\hline Presión Atmosférica & Barómetro digital & $0,5 \%$ & {$[30$ a 110$] \mathrm{kPa}$} & $80 \mathrm{kPa}$ & $1 \mathrm{kPa}$ \\
\hline Intensidad Corriente & Efecto Hall & $3 \%$ & {$[0$ a 100$] \mathrm{A}$} & $100 \mathrm{~A}$ & $0,1 \mathrm{~A}$ \\
\hline
\end{tabular}

Fuente: Autores.

Los gráficos de residuales (Fig. 12) confirman la variabilidad de la muestra, lo que indica que se trabajaron datos dentro del rango de confiabilidad en la misma. De igual forma, el coeficiente de determinación está por encima del $99 \%$ con una confianza del 95,45\%, indicando una clara relación de las variables y su capacidad de describir la razón de cambio de la magnitud de flujo de aire respecto a la señal generada por el sensor de flujo $\mathrm{BOSCH}$ 22680-7J600.

Por otra parte, aplicando la metodología descrita, se ha realizado el cálculo de incertidumbre de todos los sensores implementados en el banco de prueba. Inicialmente se obtuvieron los parámetros descritos en la tabla 5, con base en la información suministrada en las especificaciones del fabricante, los valores de referencia de libros y los catálogos de instrumentos.

La clase de exactitud fue determinada por datos del fabricante. Para los valores de resolución y rango de medición, se tuvieron en consideración especificaciones de catálogos y manuales de los dispositivos implementados en la cadena de medida, que se resumen en la tabla 5. Determinando la incertidumbre combinada y el valor de se obtuvieron los factores de cobertura asociado al nivel de confianza del 95,45\% establecido, cuyos resultados se resumen en la tabla 6 . De esta manera, se determinó el valor de incertidumbre que acota el error absoluto asociado a la medición de los instrumentos, siendo aproximados de manera conservativa. 
Tabla 6. Análisis de Incertidumbre de los Sensores de Medida.

\begin{tabular}{|c|c|c|c|c|c|c|c|c|}
\hline Variable & $E M P$ & $u_{1 B}(p)$ & $u_{2 B}(p)$ & $u_{c}(p)$ & $\beta$ & $k(p)$ & $U_{p}$ & $U_{p}$ \\
\hline Temperatura $\left[{ }^{\circ} \mathrm{C}\right]$ & 1,570 & 0,906 & 0,289 & 0,951 & 0,222 & 1,894 & 1,802 & $\approx 2$ \\
\hline Presión $[\mathrm{kPa}]$ & 0,600 & 0,346 & 0,058 & 0,351 & 0,500 & 1,786 & 0,627 & $\approx 1$ \\
\hline Flujo Másico $[\mathrm{kg} / \mathrm{hr}]$ & 2,250 & 1,299 & 0,029 & 1,299 & 0,914 & 1,651 & 2,146 & $\approx 3$ \\
\hline Régimen de Giro $[\mathrm{RPM}]$ & 2,998 & 1,731 & 0,289 & 1,755 & 0,500 & 1,786 & 3,135 & $\approx 3$ \\
\hline Humedad [\%HR] & 2,000 & 1,155 & 0,289 & 1,190 & 0,333 & 1,856 & 2,210 & $\approx 3$ \\
\hline Presión Atm. $[\mathrm{kPa}]$ & 0,400 & 0,231 & 0,289 & 0,370 & 0,429 & 1,818 & 0,672 & $\approx 1$ \\
\hline I. de Corriente $[\mathrm{A}]$ & 3,000 & 1,732 & 0,029 & 1,732 & 0,935 & 1,652 & 2,862 & $\approx 3$ \\
\hline
\end{tabular}

Fuente: Autores.

\section{Conclusiones}

En el desarrollo de la cadena de medida expuesta en este trabajo se evidencia que la totalidad de los sensores implementados fueron aislados eléctricamente, se linealizaron y su señal fue convertida utilizando la estructura de acondicionamiento de señales para las variables adquiridas en el Banco de Prueba de Motores Diésel Monocilíndricos [19]. Una gran cantidad de sensores utilizados requirieron aumentar la magnitud de la señal a niveles suficientemente altos para su registro, por lo que se aplicó un proceso de amplificación; y otros se requirió un proceso de atenuación para disminuir la magnitud de la señal de salida, lo que confirma la variabilidad en los tipos de acondicionamiento de señales adoptados en cualquier cadena de medición.

A través de un análisis de datos tomados durante el proceso de caracterización, se realizó una etapa de filtrado en donde el $13 \%$ de los datos de señales del sensor de presión y el $18 \%$ de los datos de señales del sensor de flujo de aire fueron descartados por ser valores atípicos, atribuidos a ruidos y perturbaciones en la señal durante el proceso de medición. En el proceso se evidenció que la cantidad de datos atípicos no era proporcional a las variaciones de la carga a las que fue sometido cada sensor, lo cual descarta cualquier tipo de tendencia no identificada.

Analizando los gráficos de los residuos obtenidos en el proceso de caracterización de los sensores de presión PSA-CO1 y flujo de aire BOSCH 226807J600 se logró identificar que la variabilidad máxima de la muestra fue del $22 \%$ y $5 \%$ respectivamente, permitiendo inferir que la ecuación característica que describe la razón de cambio del sensor de presión se ajusta de mejor manera a los valores de la muestra.

A partir de los resultados se ha evidenciado que 2 de los 7 sensores utilizados en la cadena de medida, tenían valores de incertidumbre menores a la unidad de medida, lo que garantiza pequeñas desviaciones respecto al valor nominal. De los 7 tipos de instrumentos de medida implementados, el sensor régimen de giro presentó el valor más alto de incertidumbre. Sin embargo, este valor se ajusta a los errores permitidos en las mediciones de RPM, los cuales oscilan un $5 \%$ su valor nominal.
El software de adquisición desarrollado cuenta con una ventana principal donde se visualizar la magnitud física de los 7 sensores ubicados en los diferentes subsistemas del motor. El programa es capaz de almacenar información a lo largo de cualquier intervalo de tiempo establecido con una frecuencia de adquisición mínima de una milésima de segundo; generando un reporte a partir de esta información.

Cabe destacar que los sensores utilizados para medir 6 de las variables poseen un tiempo de respuesta promedio de $20 \mathrm{~ms}$, siendo esta una característica determinante para realizar pruebas en régimen dinámico, aunque para el presente caso de estudio, la condición de operación del motor es en estacionaria [20]. Sin embargo, el banco de pruebas instrumentado cuenta con un motor de régimen estacionario, por lo que el restante de los sensores con un tiempo de respuesta relativamente alto $(1,4 \mathrm{~s})$, satisfacen de igual manera los requerimientos de la cadena de medida.

\section{REFERENCIAS}

[1] J. Duarte, G. Amador, J. Garcia, A. Fontalvo, R. V. Padilla, M. Sanjuan and A. G. Quiroga, "Auto-ignition control in turbocharged internal combustion engines operating with gaseous fuels," Energy, vol. 71, pp. 137-147, Jul. 2014. Doi: https://doi.org/10.1016/J.ENERGY.2014.04.040

[2] J. Duarte, J. Garcia, J. Jiménez, M. E. Sanjuan, A. Bula and J. González, "Auto-Ignition Control in Spark-Ignition Engines Using Internal Model Control Structure," $J$. Energy Resour. Technol., vol. 139, no. 2, pp. 022201, Jul. 2016. Doi: https://doi.org/10.1115/1.4034026

[3] X. Li, Z. Xu, C. Guan and Z. Huang, "Impact of exhaust gas recirculation (EGR) on soot reactivity from a diesel engine operating at high load," Appl. Therm. Eng., vol. 68, no. 1-2, pp. 100-106, Jul. 2014. Doi: https://doi. org/10.1016/J.APPLTHERMALENG.2014.04.029

[4] J. Fu, G. Zhu, F. Zhou, J. Liu, Y. Xia and S. Wang, "Experimental investigation on the influences of exhaust gas recirculation coupling with intake tumble on gasoline engine economy and emission performance," Energy Conversion and Management, vol. 127, pp. 424-436, Nov. 2016. Doi: https://doi.org/10.1016/j.enconman.2016.09.033

[5] G. Amador, J. D. Forero, A. Rincón, A. Fontalvo, A. Bula, R. V. Padilla and W. Orozco, "Characteristics of AutoIgnition in Internal Combustion Engines Operated With Gaseous Fuels of Variable Methane Number," J. Energy Resour. Technol., vol. 139, no. 4, p. 042205, Mar. 2017. Doi: https://doi.org/10.1115/1.4036044 
[6] R. Finesso, G. Hardy, C. Maino, O. Marello and E. Spessa, "A New Control-Oriented Semi-Empirical Approach to Predict Engine-Out NOx Emissions in a Euro VI 3.0 L Diesel Engine," Energies, vol. 10, no. 12, Nov. 2017. Doi: https:// doi.org/10.3390/en10121978

[7] J. Asprion, O. Chinellato and L. Guzzella, "Optimisation-oriented modelling of the NOx emissions of a Diesel engine," Energy conversion and management, vol. 75, pp. 61-73, Nov. 2013. Doi: https://doi.org/10.1016/j.enconman.2013.05.039

[8] U. Asad, R. Kumar, X. Han and M. Zheng, "Precise instrumentation of a diesel single-cylinder research engine," Measurement, vol. 44, no. 7, pp. 1261-1278, Aug. 2011. Doi: https://doi.org/10.1016/J.MEASUREMENT.2011.03.028

[9] H. Taghavifar, H. Taghavifar, A. Mardani, A. Mohebbi and S. Khalilarya, "A numerical investigation on the wall heat flux in a DI diesel engine fueled with n-heptane using a coupled CFD and ANN approach," Fuel, vol. 140, pp. 227-236, Jan. 2015. Doi: https://doi.org/10.1016/j.fuel.2014.09.092

[10] J. Chang, O. Güralp, Z. Filipi, D. Assanis, T. W. Kuo, P. Najt and R. J. Rask, "New Heat Transfer Correlation for an HCCI Engine Derived from Measurements of Instantaneous Surface Heat Flux," SAE Tech. Pap. 2004-01-2996, pp. 1576-1593, Oct. 2004. Doi: https://doi. org/10.4271/2004-01-2996

[11] M. Yao, H. Wang, Z. Zheng and Y. Yue, "Experimental study of n-butanol additive and multi-injection on HD diesel engine performance and emissions," Fuel, vol. 89, no. 9, pp. 2191-2201, Apr. 2010. Doi: https://doi.org/10.1016/j. fuel.2010.04.008

[12] M. Reyes, A. Melgar, A. Pérez and B. Giménez, "Study of the cycle-to-cycle variations of an internal combustion engine fuelled with natural gas/hydrogen blends from the diagnosis of combustion pressure," international journal of hydrogen energy, vol. 38, no. 35, pp. 15477-15487, Nov. 2013. Doi: https://doi.org/10.1016/j.ijhydene.2013.09.071

[13] P. A. A. Montoya, J. L. M. Bastidas y E. I. Ortega, "Cobertura máxima de redes de sensores inalámbricos para un sistema de gestión de energía en hogares inteligentes," INGE CUC, vol. 12 , no. 2, pp. 68-78, Dic. 2016. Doi: http:// dx.doi.org/10.17981/ingecuc.12.2.2016.07

[14] H. Liu, J. Ma, L. Tong, G. Ma, Z. Zheng and M. Yao, "Investigation on the potential of high efficiency for internal combustion engines," Energies, vol. 11, no. 3, pp. 513, Feb. 2018. Doi: https://doi.org/10.3390/en11030513

[15] D. Ashlock y A. Warren, Guía de acondicionamiento de señales para ingenieros. US: National Instruments.

[16] J. R. Lajara, y J. Pelegrí Sebastiá, LabVIEW: entorno gráfico de programación, 2a ed. Barcelona, España: Marcombo, 2011.

[17] R. W. Herschy, "The uncertainty in a current meter measurement," Flow Meas. Instrum., vol. 13, no. 5-6, pp. 281-284, Dec. 2002. Doi: https://doi.org/10.1016/S09555986(02)00047-X

[18] D. S. Moore, G. P. McCabe and B. A. Craig, Introduction to the Practice of Statistics, 9th Ed., NK, USA: Macmillan Learning, 2017.

[19] H. N. Pallares, S. V. Acosta, J. E. D. Forero y A. R. Montenegro, "Implementación de un banco para pruebas en motor Diésel monocilíndrico con aplicaciones investigativas," Scientia et technica, vol. 22, no. 4, pp. 330-340, Dic. 2017.

[20] J. D. Forero, W. G. Estrada y J. S. Guerrero. "Desarrollo de una metodología para la predicción del volumen real en la cámara de combustión de motores diésel utilizando elementos finitos", INGE CUC, vol. 14, no. 1, pp. 122-132, May. 2018. Doi: https://doi.org/10.17981/ingecuc.14.1.2018.11
Jorge Duarte Forero es Ingeniero Mecánico egresado de la Universidad del Atlántico (Colombia) y Doctor en Ingeniería Mecánica de la misma alma máter, con Magister en Ingeniería Mecánica de la Universidad del Norte (Colombia). Sus intereses son el modelado de sistemas termodinámicos y el modelado a través de paquetes de Ingeniería Asistida por Computador (Computer-Aided Engineering - CAE). Es investigador asociado de Colciencias y profesor asociado en la Universidad del Atlántico (Colombia). https://orcid.org/0000-0001-7345-9590

Kevin Berrio Orozco es Ingeniero Mecánico egresado de la Universidad del Atlántico (Colombia). Sus intereses son la instrumentación industrial y el modelado a través de paquetes de Ingeniería Asistida por Computador (Computer-Aided EngineeringCAE). https://orcid.org/0000-0003-3174-8276

Ariel Guzmán Fruto es Ingeniero Mecánico egresado de la Universidad del Atlántico (Colombia). Sus intereses son la instrumentación industrial y el modelado a través de paquetes de Ingeniería Asistida por Computador (Computer-Aided EngineeringCAE). https://orcid.org/0000-0002-1353-2714 